Journal of Applied Pharmaceutical Science Vol. 6 (06), pp. 066-072, June, 2016

Available online at http://www.japsonline.com

DOI: $10.7324 / J A P S .2016 .60612$

ISSN 2231-3354 (cc) BY-NC-SA

\title{
Sodium Butyrate Attenuates Nephrotoxicity Induced by Tamoxifen in Rats
}

\author{
Hanan Saleh*, Basma Mohamed, Mohamed-Assem S. Marie \\ Zoology Department, Faculty of Science, Cairo University, Giza, Egypt.
}

\begin{tabular}{l} 
ARTICLE INFO \\
\hline Article history: \\
Received on: $15 / 04 / 2016$ \\
Revised on: $21 / 05 / 2016$ \\
Accepted on: 09/06/2016 \\
Available online: $28 / 06 / 2016$ \\
\hline Key words: \\
Tamoxifen; sodium butyrate; \\
kidney; oxidative stress.
\end{tabular}

\begin{abstract}
Tamoxifen (TAM) is a hormonal selective estrogen modulator used in the prevention and treatment of breast cancer. It associated with increased in the oxidative stress in cells leading to tissue injury. Sodium butyrate $(\mathrm{NaBu})$ increased the glutathione redox system and diminished the oxidative stress. The purpose of this study was to elucidate the ameliorative effect of $\mathrm{NaBu}$ against $\mathrm{TAM}$-induced kidney injury by reducing the generation of oxidative stress. Rats were divided mainly into four group as follow: control, rats received saline for 14 days orally then saline intraperitoneally (i.p.) for 7 days, $\mathrm{NaBu}$, rats received saline orally for 14 days, $\mathrm{NaBu}$ $(300 \mathrm{mg} / \mathrm{kg}$ ) (i.p.) for 7 days, TAM, rats received TAM $(40 \mathrm{mg} / \mathrm{kg}$ ) orally for 14 days, then saline (i.p.) for 7 days, (TAM-NaBu) rats received TAM orally for 14 days, $\mathrm{NaBu}$ (i.p.) for 7 days. Kidney injury followed by TAM treatment was assessed by the elevation in the levels of creatinine, urea, uric acid and MDA and reduction in some oxidative biomarkers, in addition to the abnormal architecture of the kidney. Conversely, Administration of sodium butyrate could ameliorate all of these damaging effects in the antioxidant system in the TAM-treated group. NaBu affords significant increments in the antioxidant enzymes. In addition, it has the therapeutic capacity to protect the kidney from the oxidative stress induced by TAM through improving the kidney function and diminishing the free radicals. Supplementation of $\mathrm{NaBu}$ could be useful in alleviating TAM-induced kidney injury.
\end{abstract}

\section{INTRODUCTION}

Tamoxifen (TAM) is the most recommended breast anticancer oral medication for prevention and treatment of breast cancer for both men and women (Shukla et al., 2016). Tamoxifen is a hormonal selective estrogen modulator and its Pharma-cological effects are based on its binding to estrogen receptors and suppressing epithelial proliferation of different cell types that lack estrogen receptor (McDonnell, 1999; Johnston 2005). While, TAM could reduce breast cancer disease in highrisk women by $49 \%$, a number of studies indicated the TAM caused carcino-genic and genotoxic effects through TAM metabolites by pairing with Estrogen Receptors (ERs) (Lippman and Brown, 1999).

* Corresponding Author

Email: hebead@sci.cu.edu.eg
In addition, TAM has an important role in inducing endometrial and liver cancer, thromboembolic disorders in rats and humans (Kärki et al., 2000). The toxicity of TAM concerning multiple organs and tissues is well-established. This toxicity has been attributed to the reduction of the hexose monophosphate shunt and the treatment with TAM increasing the incidence of oxidative stress in cells leading to tissue injury (Tabassum et al., 2007). Generally, the kidneyis considered as the vital organ to eliminate drugs, toxins and endogenous metabolites (Torres, 2008). Previously, it was reported that carcinogenicity of TAM is attributed to its metabolites covalently bind to DNA, resulting in DNA adduct formation in the kidney (Li et al., 1997). In addition, Antineoplastic agents or adjuvant treatments including TAM are directly caused a reduction in renal functions (Kintzel and Dorr 1995). It was suggested that oxidative stress might trigger the pathogenesis of TAM-induced toxicity (Dragan et al., 1996). 
Zuhair (2011), elucidated the possible mechanism of TAM-induced nephrotoxicity. He reported that nephrotoxicity might be due to the Reactive oxygen species (ROS) produced by TAM could damage the glutathione redox system of the kidney and subsequently, induce free radicals production in renal tissue.

Furthermore, ROS generated by TAM, exceeding the antioxidant capacity so triggering cell injury and decreases its ability to detoxify ROS (Zuhair 2011). ROS such as superoxide anions $\left(\mathrm{O}_{2}^{-}\right)$, hydroxyl radical $(\mathrm{OH})$, and hydrogen peroxide $\left(\mathrm{H}_{2} \mathrm{O}_{2}\right)$ are important mediators of tissue injury (Saad and AlRikabi 2002).ROS generated in the cells by TAM can be scavenged by an antioxidant defense system, such as the glutathione redox system which includes, reduced glutathione (GSH), glutathione peroxidase (GPx), and glutathione-Stransferase (GST) (Halliwell 2007). Antioxidants play an essential role in defending the body from oxidative stress at a cellular level. Therefore, improving the antioxidant system is the principle target to diminish the nephrotoxicity induced by TAM. Several natural products and some synthetic substances display antioxidants impact that affords protection against free radical induced renal damage.

Sodium butyrate is a natural 4-carbon short chain fatty acid that synthesized by the microflora in the colon and functions as the primary source of energy for colonocytes (Liu et al., 2016). In addition, it is known as histone deacetylase inhibitors. Butyrate inhibits cell proliferation and causes differentiation in many tumorous and non-tumorous cells (Niki et al., 1999). It was assumed that the modulation of the glutathione transferase gene may protect cells from genotoxic carcinogens, such as $\mathrm{H}_{2} \mathrm{O}_{2}$ and 4hydroxynonenal (Canani, Di Costanzo, and Leone 2012).The mechanism proposed of sodium butyrate is the transcriptional upregulation of detoxifying enzymes, such as GST. Recent studies (Yang et al., 2014; Liu et al., 2016) indicated that sodium butyrate exerts anti-inflammatory effect is through the inhibition of the expression of inflammatory mediators such as nuclear factorkappa B (NF-кB), tumor necrosis factoral pha (TNF- $\alpha$ ) and interferon-gamma (IFN- $\gamma$ ). The ability of sodium butyrate to exert its antioxidant effect by affecting DNA repair systems and activities of enzymatic or non-enzymatic antioxidants (Hamer $e t$ al., 2009).

Nonetheless, there is negligible literature on the impact of sodium butyrate using an in vivo rat model. Therefore, the aim of this study was to investigate the therapeutic effect of sodium butyrate against TAM-induced nephrotoxicity in rat.

\section{MATERIAL AND METHODS}

\section{Chemicals and Reagents}

Nolvadex (tamoxifen citrate $40 \mathrm{mg}$ ) was obtained from AstraZeneca (Egypt). Sodium butyrate was purchased from Sigma-Aldrich (St. Louis, MO, USA). Kits for all biochemical parameters were purchased from Biodiagnostic Company diagnostic and research reagents (Dokki, Giza, Egypt).

\section{Experimental Animals}

The experimental animals used in this study were male albino rats Rattus norvegicus weighing $(250-300 \pm 5 \mathrm{~g})$. The animals were purchased from National Research Center (NRC, Giza, Egypt). Animals were given food and water ad libitum. Rats were maintained in a friendly environment with a $12 \mathrm{~h} / 12 \mathrm{~h}$ lightdark cycle at room temperature $\left(22{ }^{\circ} \mathrm{C}-25{ }^{\circ} \mathrm{C}\right)$. Rats were acclimatized to laboratory conditions for 7 days before commencement of the experiment.

\section{Ethical Consideration}

Experimental protocols and procedures used in this study were approved by the Cairo University, Faculty of Science, Institutional Animal Care and Use Committee (IACUC) (Egypt) (CUFS/F/PHY/45/14). All the experimental procedures were carried out in accordance with international guidelines for the care and use of laboratory animals.

\section{Experimental Design}

Rats were divided into 4 groups randomly ( $\mathrm{n}=6$ / group) as follows:

Group 1: Control group, treated orally saline for 14 days then injected intraperitoneally (i.p.) with saline for 7 days. Group 2: NaBu group, received orally saline for 14 days then received an (i.p.) dosage of sodium butyrate $(300 \mathrm{mg} / \mathrm{kg}$ ) for 7 days.

Group 3: TAM group, treated with an oral dosage of TAM ( $40 \mathrm{mg} / \mathrm{kg}$ b. wt.) orally for 14 days followed i.p. administration of saline for 7 days.

Group 4: TAM-NaBu group, rats were treated with TAM orally for 14 days followed by sodium butyrate (i.p.) for 7 days. The doses of TAM and $\mathrm{NaBu}$ were chosen from previously published reports (Hashiba et al., 2006; Hemieda 2007; Ferrante et al., 2003).

\section{Animal Handling}

At the end of the experiment, rats were euthanized under anesthesia; their blood samples were collected and centrifuged at (3000 r.p.m, 15 minutes). Serum was stored at $-20^{\circ} \mathrm{C}$ until used for biochemical assays.

\section{Kidney Homogenate Preparation}

The left kidney was homogenized $(10 \% \mathrm{w} / \mathrm{v})$ in ice-cold $0.1 \mathrm{M}$ Tris- $\mathrm{HCl}$ buffer $(\mathrm{pH}=7.4)$. The homogenate was centrifuged at (3000 r.p.m, 15 minutes) at $4^{\circ} \mathrm{C}$ and the resultant supernatant was used for the biochemical analyses.

\section{Biochemical Analysis of Kidney Function Test}

The levels of renal function markers included creatinine, urea, and uric acid were determined using the appropriate commercially kits (Bio-Diagnostic, Dokki, Giza, Egypt).Serum urea and uric acid were measured according to (Tietz 1995), 
serum creatinine was measured according to the method of (Tietz and Andresen 1986).

\section{Kidney Oxidative Stress Assessment}

Malondialdehyde (MDA) (Ohkawa et al.,1979), reduced glutathione (GSH) (Beutler, Duron, and Kelly 1963), catalase (CAT) (Aebi 1984), superoxide dismutase (SOD) (Nishikimi et al., 1972), glutathione peroxidase (GPx) (Paglia and Valentine 1967), glutathione-S-transferase (GST) (Habig, Pabst, and Jakoby 1974) and nitric oxide (NO) (Montgomery and Dymock 1961), were determined using Biodiagnostic assay kits according to the manufacturer's instructions (Bio-Diagnostic, Dokki, Giza, Egypt).

\section{Histopathological Preparation.}

The right kidney was fixed in $10 \%$ neutral buffered formalin solution and embedded in paraffin wax blocks. Sections of $5 \mu \mathrm{m}$ thickness were stained with hematoxylin and eosin (H\&E) then examined under light microscope for determination of pathological changes (Meyer 1903).

\section{Statistical Analysis}

Data are expressed as mean $\pm \mathrm{SE}$ of six rats in each group. Treated groups are compared with control and TAM group by using Unpaired Student's t-test ${ }^{*} P<0.05,{ }^{* *} P<0.01$ vs control group, ${ }^{\#} P<0.05$; $P<0.01$ vs TAM. Graph Pad Prism 6 software (Graph PadSoftware, Inc., San Diego, USA) was used for statistical analysis.

\section{RESULTS}

\section{Kidney function tests}

The nephrotoxicity induced by TAM was established by the oral administration of TAM for 14 days. Revealed nephrotoxicity was characterized by a significant increase $(P<0.01)$ in serum creatinine, urea, and uric acid in TAM-treated rats as compared to the control group. On the contrary, treatment with $\mathrm{NaBu}$ reversed TAM-induced kidney damage by reducing the levels of creatinine, urea, and uric acid significantly $(P<0.01)$ compared with the TAM group (Figure 1).
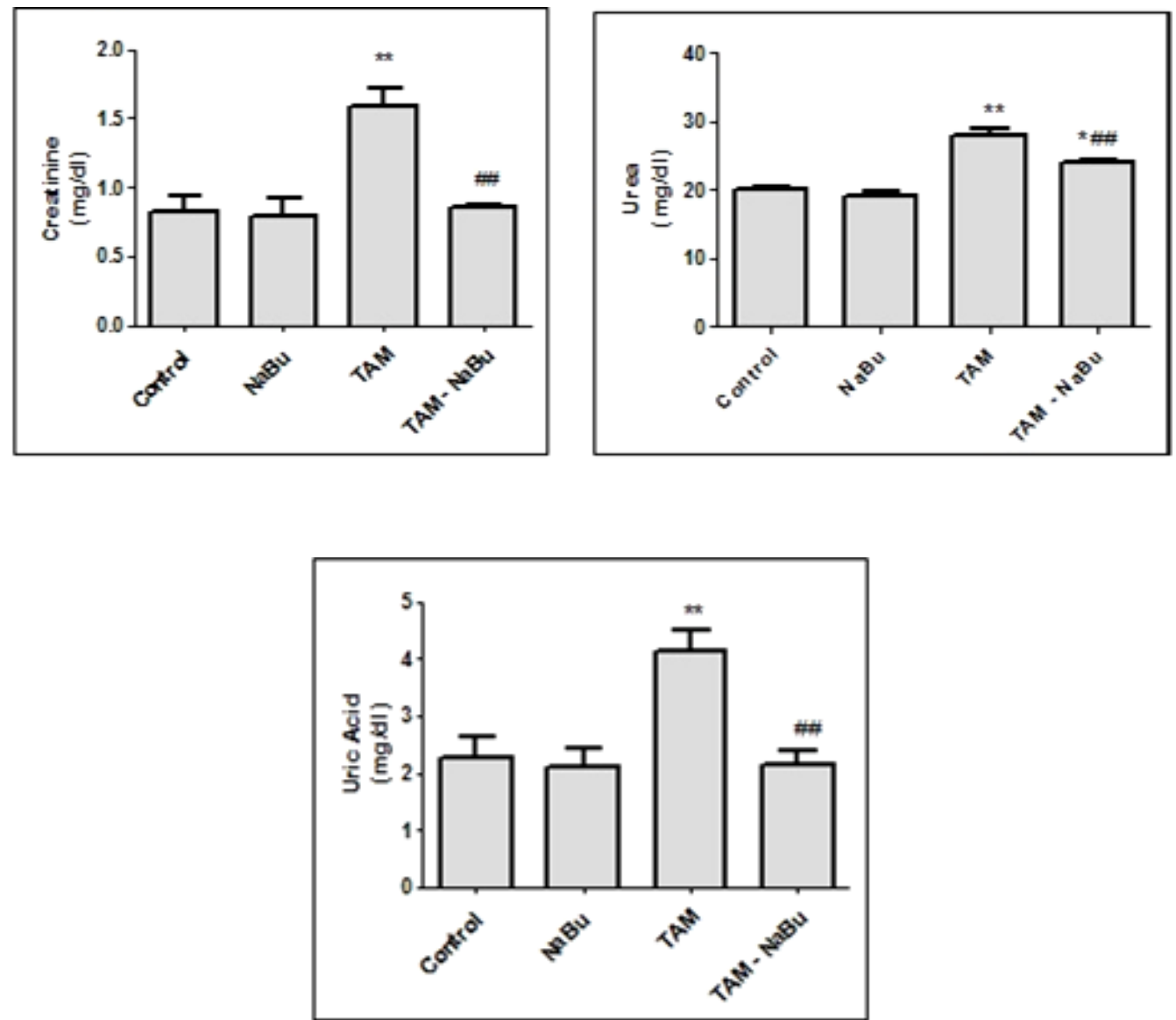

Fig. 1: Effect of $\mathrm{NaBu}$ on serum urea, uric acid and creatinine of rat treated with TAM. Values are expressed as means $\pm S E(n=6)$. Significant difference is indicated by ${ }^{*} P<0.05,{ }^{* *} P<0.01$ when compared with control group. Significant difference is indicated by ${ }^{\#} P<0.05,{ }^{\# \#} P<0.01$ when compared with TAM group. 


\section{Renal Oxidative stress biomarkers}

Treatment with TAM orally for 14 days caused a significant increase $(P<0.01)$ in MDA and NO concentration of renal tissues, as compared with the control group. TAM weakened the antioxidant defense system by decreasing significantly the GSH $(P<0.05)$, CAT, SOD, GPx, GST $(\mathrm{P}<0.01)$ activities in the renal tissues when compared with the corresponding values of the control group. Treatment with $\mathrm{NaBu}$ (TAM-NaBu) was effective in the inhibition of oxidative damage induced by TAM, where it reduced the MDA and NO levels significantly $(P<0.01)$. Moreover, $\mathrm{NaBu}$ significantly increases $(P<0.01)$ the renal antioxidant activities such as GSH, CAT, SOD and GPx as compared with TAM group (Figure 2).
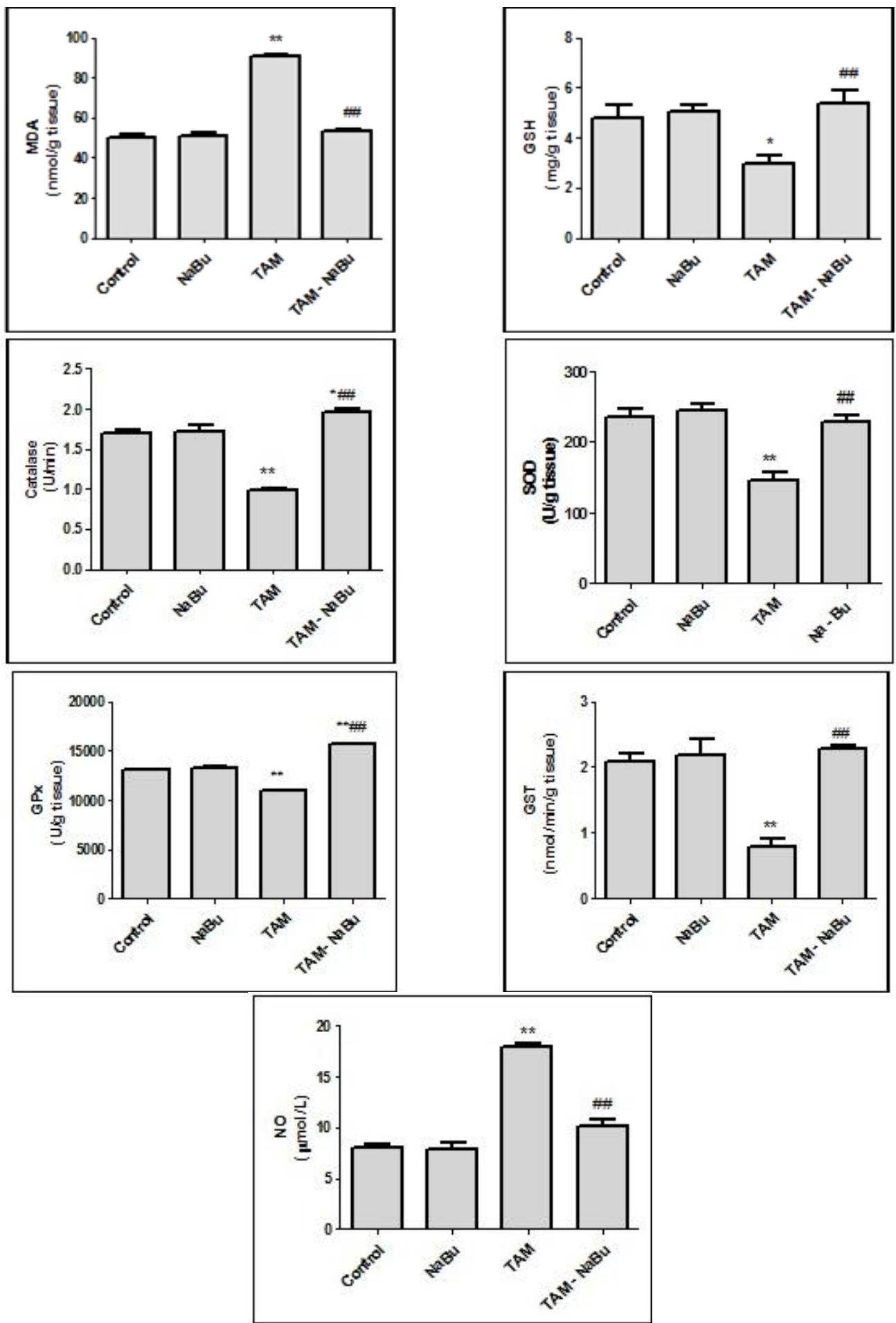

Fig. 2: Effect of $\mathrm{NaBu}$ on enzymatic and non-enzymatic antioxidant in kidney of rat treated with TAM. Values are expressed as means $\pm \mathrm{S} E(\mathrm{n}=6$ ). Significant difference is indicated by ${ }^{*} P<0.05,{ }^{* *} P<0.01$ when compared with control group. Significant difference is indicated by ${ }^{\#} P<0.05,{ }^{\# \#} P<0.01$ when compared with TAM group. 
A

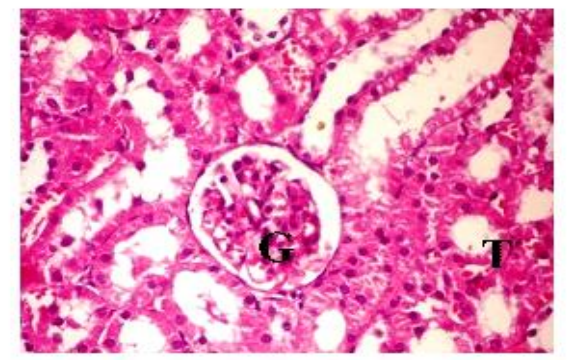

$\mathrm{C}$

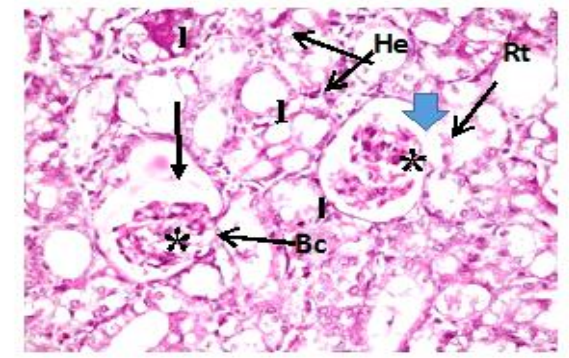

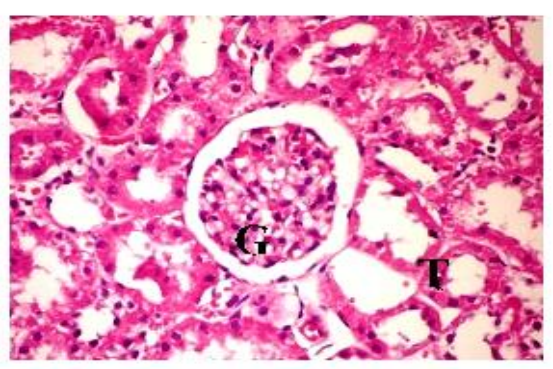

B

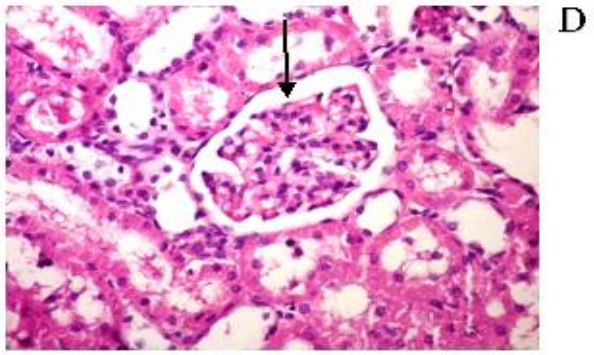

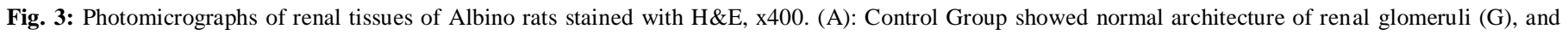

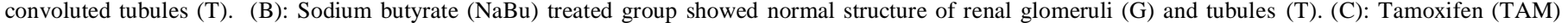

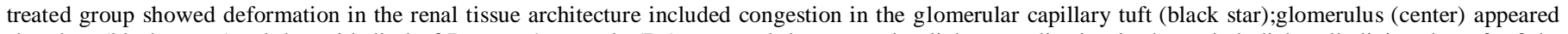

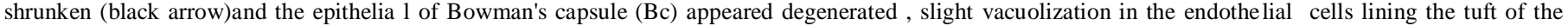

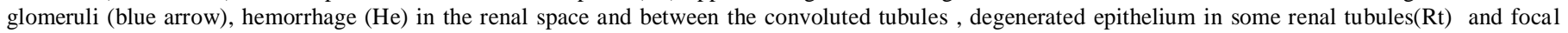

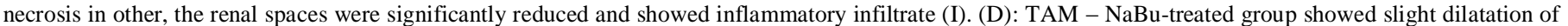
renal tubules (arrow), most of these tubules restore their normal architecture.

\section{Histopathological Examination of Kidney}

The kidney tissue of the control group (Figure 3 A) and $\mathrm{NaBu}$ group (Figure $3 \mathrm{~B}$ ) show the normal architecture of health tissue including the normal structure of renal glomeruli and renal tubules. Oral administration of TAM (Figure $3 \mathrm{C}$ ) reflects the complications in the architecture of kidney. Where, congestion in the glomerular capillary tuft, slight vacuolization in the endothelial cells lining the tuft of the glomeruli, wide congestion in the glomerular capillary mass, inflammatory cells infiltration, the absence of epithelial cells of renal tubules and deformation in the renal tissue architecture were observed. While, the administration of $\mathrm{NaBu}$ as the treatment mediator could restore the healthy morphological and structural shape of the kidney, where the corpuscles and tubules appeared to be similar to those of the control group excepts light dilatation of renal tubules (Figure $3 \mathrm{D}$ ). Data from the histological study supports the biochemical analyses and confirmed the TAM has antinephrotoxic activity.

\section{DISCUSSION}

The incidence of kidney failure is mostly associated with the chemotherapy-induced renal dysfunction (Liangos et al., 2006). Kidney damage is irreversible and may lead to the development of end-stage renal disease (Hamad et al., 2015). Lien et al. (1991) clarified that the highest levels of TAM and its metabolites were observed in lung, liver, and kidney. There are very few researchers that discuss the effect of TAM on the kidney function tests. Thus, in the present study, we measured the nitrogenous end products; creatinine, urea and uric acid which reflect the efficacy of kidney function. The present study exposed that the oral administration of TAM for 14 days caused nephrotoxicity, where, creatinine, uric acid, and urea levels were increased. These data are accordance with Ahmed et al. (2008) who mentioned that the oral TAM therapy is associated with the damage of kidney function tests which is connected with impairment to the vasculature or/and structures of the kidneys (Ahmed et al., 2008). Oxidative stress is considered as the important factor in the development of a variety of human complications that result from over production of ROS and impairment in the biological defense system (Tabassum et al., 2007). There is increasing evidence that TAM generates ROS production and depletion in the cellular thiol system which are important mediators of nephrotoxicity (Parlakpinar et al., 2005). Moreover, ROS interact with the renal mitochondrial membranes producing large amounts of oxygen radicals that cause deterioration of the kidney architecture (Tabassum et al., 2007). In the current study, treatment with TAM for 14 days caused renal oxidative stress by increasing the Malondialdehyde (MDA) level, as an indicator of lipid peroxidation, and altered non-enzymatic and enzymatic antioxidant systems in the renal tissues through the reduction of GSH, CAT, SOD, GPx and GST activities. In accordance with these obtained data, (El-Beshbishy 2005), reported that administration of TAM caused an elevation in lipid peroxidation which is attributed to the reduction in nicotinamide adenine dinucleotide phosphate (NADPH), which involved in the protecting against ROS inside the cells. In addition, the activities of some intracellular antioxidant enzymes decreased with the increase of lipid peroxidation level (Diplock, Rice-Evans, and 
Burdon 1994). GSH is the non-enzymatic antioxidant present in cells that have a vital role in the defense against oxidative stress induced by cell injury. Moreover, it is protecting cells against ROS. The progress of nephrotoxicity may be affected by depleting GSH activity which consequently causes an inhibition of GPx that makes the cells more susceptible to the injury occurred by oxidative stress (Brittebo et al., 1993). SOD is a specific antioxidant enzyme that dismutase $\mathrm{O}_{2}^{-}$, forming $\mathrm{H}_{2} \mathrm{O}_{2}$, which is scavenged by CAT or GPx. Both enzymes, CAT, and GPx together convert hydrogen peroxide to water (Fadillioğlu et al., 2003).GST plays a significant role in the detoxification of foreign compounds. The results of the present study demonstrate that TAM treatment causes oxidative tissue damage, as assessed by decreased GSH, CAT, SOD, GPx, GST activities in the kidney. These finding data are in line with the finding of (Tabassum et al., 2007).On the contrary, sodium butyrate treatment improved the enzymatic and non-enzymatic antioxidant enzymes in the renal tissues that protect the kidney against TAM caused nephrotoxicity. Moreover, Tamoxifen-induced mitochondrial NO that stimulates the increases in mitochondrial peroxynitrite, which causes ROS and reactive nitrogen species (RNS) (Nazarewicz et al., 2007). In the current study, the overproduction of ROS and RNS may be related to damage to vasculature or structures of the kidneys which were established by the histopathological examination of renal architecture. Where, the congestion in the glomerular capillary tuft and absence of epithelial cells of the renal tubules were reflected the severe pathological effect of TAM and consequently, altered the kidney function tests and the damage the defense system of the kidney. These data are accordance with (Ahmed et al., 2008)who mentioned that the oral TAM therapy is associated with impairment of kidney function. From all the previous results, this study suggests that the depletion of the redox system and liberation of MDA and free radicals by treatment with TAM caused an alteration in the kidney structure which consequently, affect the kidney function through elevation of the endogenous end products, creatinine, urea and uric acid in the blood. On the other hand, (Padillo et al., 2005) showed that the damage occurred in the kidney by TAM is due to impairment in the liver due to hyperbilirubinemia. Moreover, the increased level of bilirubin caused significant alterations in kidney function and damage to the renal epithelium. The previous study hypothesized that the association between the elevation in bilirubin and kidney dysfunction mainly reflects the link between serum bilirubin concentrations with nonalcoholic fatty liver disease (NAFLD) which induced by TAM therapy in more than $30 \%$ of breast cancer patients (Targher et al., 2009). Our previous study (Saleh et al., 2016) showed that the administration of TAM for 14 days caused an elevation in bilirubin level and this level was diminished by the administration of $\mathrm{NaBu}$ for 7 consecutive days. On the other hand, kidney function tests were improved in rats treated with TAM and $\mathrm{NaBu}$ as compared to the TAM treated group. The results of the current study indicated that the exogenously administered $\mathrm{NaBu}$ may reduce TAM-induced nephrotoxicity in rats. The therapeutic effect of $\mathrm{NaBu}$ may be attributed to its role to promote liver lipogenesis and prevent the progression of NAFLD which in turn decreased the bilirubin level and return the kidney function tests nearly to the normal levels (Daubioul et al., 2002; Oldfield IV and Johnson 2015).

\section{CONCLUSION}

The results of the present study revealed that the administration of TAM for 14 days caused oxidative stress mediated damage in the renal tissues. Oxidative stress generated by TAM could increase the levels of kidney function tests, MDA, and $\mathrm{NO}$ contents. In addition to the marked depletion in the antioxidant defense resulting nephrotoxicity. These alterations in the defense system affect the structure of the kidney architecture. The exogenously administered sodium butyrate may attenuate TAM-induced renal toxicity in rats. The proposed mechanism of sodium butyrate to improve the function of the kidney probably due to ameliorate the levels of creatinine, urea, and uric acid. Moreover, it could counteract the toxicity action of renal free radicals generated by TAM through the upregulation of oxidant defense system.

\section{REFERENCES} 105: $121-26$

Aebi, Hugo. Catalase in vitro. Methods in enzymology, 1984;

Ahmed, Sofia B, Bruce F Culleton, Marcello Tonelli, Scott W Klarenbach, Jennifer M MacRae, Jianguo Zhang, and Brenda R Hemmelgarn. Oral estrogen therapy in postmenopausal women is associated with loss of kidney function. Kidney international, 2008; 74: 370-76.

Beutler, Ernest, Olga Duron, and B Mikus Kelly. Improved method for the determination of blood glutathione. The Journal of laboratory and clinical medicine, 1963; 61: 882-88.

Brittebo, Eva B, Per Ola Darnerud, Catarina Eriksson, and Ingvar Brandt. Nephrotoxicity and covalent binding of 1, 1dichloroethylene in buthionine sulphoximine-treated mice. Archives of toxicology, 1993; 67: 605-12.

Canani, R Berni, Margherita Di Costanzo, and Ludovica Leone. The epigenetic effects of butyrate: potential therapeutic implications for clinical practice. Clin Epigenetics, 2012; 4: 4-4.

Daubioul, Catherine, Nicolas Rousseau, Roger Demeure, Bernard Gallez, Henryk Taper, Barbara Declerck, and Nathalie Delzenne. Dietary fructans, but not cellulose, decrease triglyceride accumulation in the liver of obese Zucker fa/fa rats. The Journal of nutrition, 2002; 132: 967-73.

Diplock, Anthony T, Catherine A Rice-Evans, and Roy H Burdon. Is there a significant role for lipid peroxidation in the causation of malignancy and for antioxidants in cancer prevention? Cancer research, 1994; 54: 1952s-56s.

Dragan, YP, S Fahey, E Nuwaysir, C Sattler, K Babcock, J Vaughan, R McCague, VC Jordan, and HC Pitot. The effect of tamoxifen and two of its non-isomerizable fixed-ring analogs on multistage rat hepatocarcinogenesis. Carcinogenesis, 1996; 17: 585-94.

El-Beshbishy, Hesham A. Hepatoprotective effect of green tea (Camellia sinensis) extract against tamoxifen-induced liver injury in rats. BMB Reports, 2005; 38: 563-70.

Fadillioğlu, Ersin, Hasan Erdoğan, Sadik Söğüt, and Irfan Kuku. Protective effects of erdosteine against doxorubicin-induced cardiomyopathy in rats. Journal of Applied Toxicology, 2003; 23: 71-74.

Ferrante, Robert J, James K Kubilus, Junghee Lee, Hoon Ryu, Ayshe Beesen, Birgit Zucker, Karen Smith, Neil W Kowall, Rajiv R Ratan, and Ruth Luthi-Carter. Histone deacetylase inhibition by sodium 
butyrate chemotherapy ameliorates the neurodegenerative phenotype in Huntington's disease mice. The Journal of neuroscience, 2003;23:9418-27.

Habig, William H, Michael J Pabst, and William B Jakoby. Glutathione S-transferases the first enzymatic step in mercapturic acid formation. Journal of biological Chemistry, 1974; 249: 7130-39.

Halliwell, Barry. Biochemistry of oxidative stress. Biochemical Society Transactions, 2007; 35: 1147-50.

Hamad, Rania, Calpurnia Jayakumar, Punithavathi Ranganathan, Riyaz Mohamed, Mahmoud MI El-Hamamy, Amina A Dessouki, Abdelazim Ibrahim, and Ganesan Ramesh. Honey feeding protects kidney against cisplatin nephrotoxicity through suppression of inflammation. Clinical and Experimental Pharmacology and Physiology, 2015; 42: 843-48.

Hamer, Henrike M, Daisy MAE Jonkers, Aalt Bast, Steven ALW Vanhoutvin, Marc AJG Fischer, Andrea Kodde, Freddy J Troost, Koen Venema, and Robert-Jan M Brummer. Butyrate modulates oxidative stress in the colonic mucosa of healthy humans. Clinical Nutrition, 2009; 28: 88-93.

Hashiba, Masamichi, Toshihiko Kasahara, Sung Yeon Kim, Shinya Shibutani, and Masakuni Degawa. DNA damage and altered gene expression of enzymes for metabolism and DNA repair by tamoxifen and toremifene in the female rat liver. Cancer science, 2006; 97: 468-77.

Hemieda, Faried Abdel-Kader El-Sayed. Influence of gender on tamoxifen-induced biochemical changes in serum of rats. Molecular and cellular biochemistry, 2007; 301: 137-42.

Johnston, Stephen RD. Selective oestrogen receptor modulators and downregulators for breast cancer-have they lost their way? Breast Cancer Res, 2005; 7: 119-30.

Kärki, Annukka, Eero Mäntylä, Yrjö Hirsimäki, Stefan Karlsson, Sakari Toikkanen, and Pirkko Hirsimäki. Comparison of the effects of tamoxifen and toremifene on rat hepatocarcinogenesis. Archives of toxicology, 2000; 74: 249-56.

Kintzel, Polly E, and Robert T Dorr. Anticancer drug renal toxicity and elimination: dosing guidelines for altered renal function. Cancer treatment reviews, 1995; 21: 33-64.

Li, Donghui, Yvonne Dragan, V Craig Jordan, Mianying Wang, and Henry C Pitot. Effects of chronic administration of tamoxifen and toremifene on DNA adducts in rat liver, kidney, and uterus. Cancer research, 1997; 57: 1438-41.

Liangos, Orfeas, Ron Wald, John W O’Bell, Lorilyn Price, Brian J Pereira, and Bertrand L Jaber. Epidemiology and outcomes of acute renal failure in hospitalized patients: a national survey. Clinical Journal of the American Society of Nephrology, 2006; 1: 43-51.

Lien, Ernst A, Einar Solheim, and Per M Ueland. Distribution of tamoxifen and its metabolites in rat and human tissues during steadystate treatment. Cancer research, 1991; 51: 4837-44.

Lippman, Scott M, and Powel H Brown. Tamoxifen prevention of breast cancer: an instance of the fingerpost. Journal of the National Cancer Institute, 1999; 91: 1809-19.

Liu, Jiaming, Fangyan Wang, Haihua Luo, Aihua Liu, Kangxin $\mathrm{Li}$, Cui Li, and Yong Jiang. Protective effect of butyrate against ethanolinduced gastric ulcers in mice by promoting the anti-inflammatory, antioxidant and mucosal defense mechanisms. International immunopharmacology, 2016; 30: 179-87.

McDonnell, Donald P. The molecular pharmacology of SERMs. Trends in Endocrinology \& Metabolism, 1999; 10: 301-11.

Meyer, Adolf. An attempt at analysis of the neurotic constitution. The American Journal of Psychology, 1903; 14: 90-103.

Montgomery, H, and JF Dymock. 1961. "Determination of nitrite in water." In, 414-\&. Royal soc chemistry thomas graham house, science park, milton rd, cambridge cb4 0wf, cambs, england.

Nazarewicz, Rafal R, Woineshet J Zenebe, Arti Parihar, Sarah K Larson, Enver Alidema, Jiho Choi, and Pedram Ghafourifar. Tamoxifen induces oxidative stress and mitochondrial apoptosis via stimulating mitochondrial nitric oxide synthase. Cancer research, 2007; 67: 1282-90.

Niki, Toshiro, Krista Rombouts, Pieter De Bleser, Karen De Smet, Vera Rogiers, Detlef Schuppan, Minoru Yoshida, Giulio Gabbiani, and Albert Geerts. A histone deacetylase inhibitor, trichostatin A, suppresses myofibroblastic differentiation of rat hepatic stellate cells in primary culture. Hepatology, 1999; 29: 858-67.

Nishikimi, Morimitsu, N Appaji Rao, and Kunio Yagi. The occurrence of superoxide anion in the reaction of reduced phenazine methosulfate and molecular oxygen. Biochemical and biophysical research communications, 1972; 46: 849-54.

Ohkawa, Hiroshi, Nobuko Ohishi, and Kunio Yagi. Assay for lipid peroxides in animal tissues by thiobarbituric acid reaction. Analytical biochemistry, 1979; 95: 351-58.

Oldfield IV, Dong RZ, and DA Johnson. Non-alcoholic fatty liver disease and the gut microbiota: exploring the connection. Gastro Open J, 2015; 1: 30-43.

Padillo, FJ, A Cruz, J Briceno, A Martin-Malo, C Pera-Madrazo, and A Sitges-Serra. Multivariate analysis of factors associated with renal dysfunction in patients with obstructive jaundice. British Journal of Surgery, 2005; 92: 1388-92.

Paglia, Donald E, and William N Valentine. Studies on the quantitative and qualitative characterization of erythrocyte glutathione peroxidase. The Journal of laboratory and clinical medicine, 1967; 70: 158-69.

Parlakpinar, H, S Tasdemir, A Polat, A Bay-Karabulut, N Vardi, M Ucar, and A Acet. Protective role of caffeic acid phenethyl ester (cape) on gentamicin-induced acute renal toxicity in rats. Toxicology, 2005; 207: 169-77.

Saad, Sherif Y, and Ammar C Al-Rikabi. Protection effects of taurine supplementation against cisplatin-induced nephrotoxicity in rats. Chemotherapy, 2002; 48: 42-48.

Saleh, Basma Mohamed, Mohamed-Assem S. Marie. Protective and Therapeutic Efficacy of Sodium Butyrate on Tamoxifen-Induced NonAlcoholic Fatty Liver Disease in Male Rats. Research Journal of Pharmaceutical, Biological and Chemical Sciences, 2016; 7: 951-65.

Shukla, Jaya, Amit Kumar Dinda, Abhay Krishna Srivastava, Kamna Srivastava, Bhagwant Rai Mittal, and Guru Pad Bandopadhyaya. Nanotamoxifen delivery system: Toxicity assessment after oral administration and biodistribution study after intravenous delivery of radiolabeled nanotamoxifen. World Journal of Nuclear Medicine, 2016; 15: 7 .

Tabassum, Heena, Suhel Parvez, Hasibur Rehman, Basu Dev Banerjee, Detlef Siemen, and Sheikh Raisuddin. Nephrotoxicity and its prevention by taurine in tamoxifen induced oxidative stress in mice. Human \& experimental toxicology, 2007; 26: 509-18.

Targher, Giovanni, Cortney Bosworth, Jessica Kendrick, Gerard Smits, Giuseppe Lippi, and Michel Chonchol. Relationship of serum bilirubin concentrations to kidney function and albuminuria in the United States adult population. Findings from the National Health and Nutrition Examination Survey 2001-2006. Clinical Chemistry and Laboratory Medicine, 2009; 47: 1055-62.

Tietz, Norbert W. 1995. Clinical guide to laboratory tests WB Saunders. Philadelphia, PA.

Tietz, Norbert W, and Brian D Andresen. 1986. Textbook of clinical chemistry (Saunders Philadelphia et al.).

Torres, Adriana Mónica. Renal elimination of organic anions in cholestasis. World J Gastroenterol, 2008; 14: 6616-21.

Yang, Fan, Li-Kun Wang, Xun Li, Lu-Wen Wang, Xiao-Qun Han, and Zuo-Jiong Gong. Sodium butyrate protects against toxin-induced acute liver failure in rats. Hepatobiliary \& Pancreatic Diseases International, 2014; 13: 309-15.

Zuhair, Zaina. The role of vitamin $\mathrm{C}$ in alteration of enzymes responsible of energy metabolism induced by administration of tamoxifen to mouse. Advances in Biological Chemistry, 2011; 1: 15.

\section{How to cite this article:}

Saleh H, Mohamed B, Marie MAS. Sodium Butyrate Attenuates Nephrotoxicity Induced by Tamoxifen in Rats. J App Pharm Sci, 2016; 6 (06): 066-072. 